\title{
Development of a Thin-Wall Superconducting Magnet for the Positron Spectrometer in the MEG Experiment
}

\author{
Wataru Ootani, Wataru Odashima, Satoshi Kimura, Takayuki Kobayashi, Yasuhiro Makida, Toshiya Mitsuhashi, \\ Shoichi Mizumaki, Roger Ruber, and Akira Yamamoto
}

\begin{abstract}
A thin-wall superconducting magnet was developed for the positron spectrometer in the MEG experiment. The magnet is specially designed to provide a gradient magnetic field to achieve good features of the spectrometer such as constant projected bending radius for monochromatic positrons and much quicker sweep of positrons than in the conventional uniform solenoidal field, which allows a stable operation of the spectrometer in a high rate muon beam. A high-strength aluminum-stabilized conductor was developed so as to minimize the thickness of the coil between the target and photon detector. A pair of compensation coils is implemented in the magnet to cancel stray field around the photon detector to be placed closely to the magnet. Design of the magnet and results from the excitation tests to measure performance of the magnet will be presented here.
\end{abstract}

Index Terms-Detectors, superconducting cables, superconducting magnets.

\section{INTRODUCTION}

A superconducting solenoidal magnet was developed for the COBRA (COnstant Bending RAdius) positron spectrometer in the MEG experiment, which is planned to search for the lepton flavor violating decay, $\mu^{+} \rightarrow \mathrm{e}^{+} \gamma$ at Paul Scherrer Institute (PSI) [1]. The magnet is specially designed to provide a gradient magnetic field in order to overcome some problems inevitable in a simple uniform solenoidal field. The gradient field is arranged so that the positrons from the target follow trajectories with constant projected bending radius independent of the emission angle. It allows us to easily define the momentum window of the positrons to be detected. In a simple uniform field positrons emitted close to right angle make many turns and would cause unstable operation of the drift chamber system. In the gradient field distribution, the positrons emitted close to right angle can be swept away much more quickly. The central field is $1.27 \mathrm{~T}$ at $z=0$ and slowly decreasing as $|z|$ increases.

Manuscript received October 21, 2003. This work was supported in part by a Grant-in-Aid for Scientific Research on Priority Areas (A) provided by the Ministry of Education, Culture, Sports, Science and Technology of Japan.

W. Ootani and T. Mitsuhashi are with the International Center for Elementary Particle Physics, University of Tokyo, Tokyo 113-0033, Japan (e-mail: wataru@icepp.s.u-tokyo.ac.jp).

W. Odashima, S. Kimura, T. Kobayashi, and S. Mizumaki are with the Toshiba Corporation, Yokohama 230-0045, Japan.

Y. Makida and A. Yamamoto are with the High Energy Accelerator Research Organization (KEK), Ibaraki 305-0801, Japan.

R. Ruber is with CERN, Geneva CH-1211, Switzerland.

Digital Object Identifier 10.1109/TASC.2004.829721

\section{MAGNet Design}

A cross section of the magnet is shown in Fig. 1. The magnet consists of a main superconducting magnet and a pair of compensation coils to reduce stray field around the photon detector to be placed close to the main magnet. The compensation coil is a resistive coil. Parameters of the superconducting magnet are listed in Table I. The superconducting magnet consists of five coils with three different radii; one central coil, two gradient coils, and two end coils. The end coil is separated into inner and outer parts with different current densities. The field gradient is arranged by a step-structure in the coil layout and adjusting winding density in each coil.

The superconducting magnet is conductively cooled by two mechanical refrigerators from both ends of the magnet. The refrigerator is a two-stage GM refrigerator (SHI SRDK-408D) with a cooling power of $31 \mathrm{~W}$ and $1 \mathrm{~W}$ at $40 \mathrm{~K}$ first stage and 4.2 $\mathrm{K}$ second stage, respectively. Cooling pipe for liquid nitrogen is attached on the end coil for a shorter cooling time and uniform cooling of the coils.

The layer structure in the central coil is illustrated in Fig. 2. In the central coil the conductor is densely wound edge-wise in four layers inside a $2 \mathrm{~mm}$-thick aluminum support cylinder. Because the superconducting magnet consists of separated coils with different radii, the mechanical support structure of the coils and the thermal conduction scheme between the coils were carefully designed. The energy stored in the coils would be dumped in a narrow region because the cold mass of the magnet is modest. Quench protection heaters are attached to the coils in order to avoid such a local energy dump. Pure aluminum strips with a thickness of $100 \mu \mathrm{m}$ are also attached to the surface of the coils along the magnet axis to increase thermal conductivity. Quench-back due to a relatively high external dump resistor $(2.8 \Omega)$ will also contribute to fast propagation of the quench.

Within the acceptance of the photon detector $(|\cos \theta| \leq$ 0.35 ), the thickness of the magnet is reduced down to $0.197 X_{0}$ so that the photons from the target placed at center of the magnet can traverse.

In order to realize this thickness a high-strength aluminumstabilized conductor is used for the superconducting magnet. It allows us to minimize the thickness of the support cylinder of the superconducting magnet. A cross-sectional view of the conductor is shown in Fig. 2. A copper matrix NbTi multi filamentary core wire is clad with aluminum stabilizer. The aluminum- 

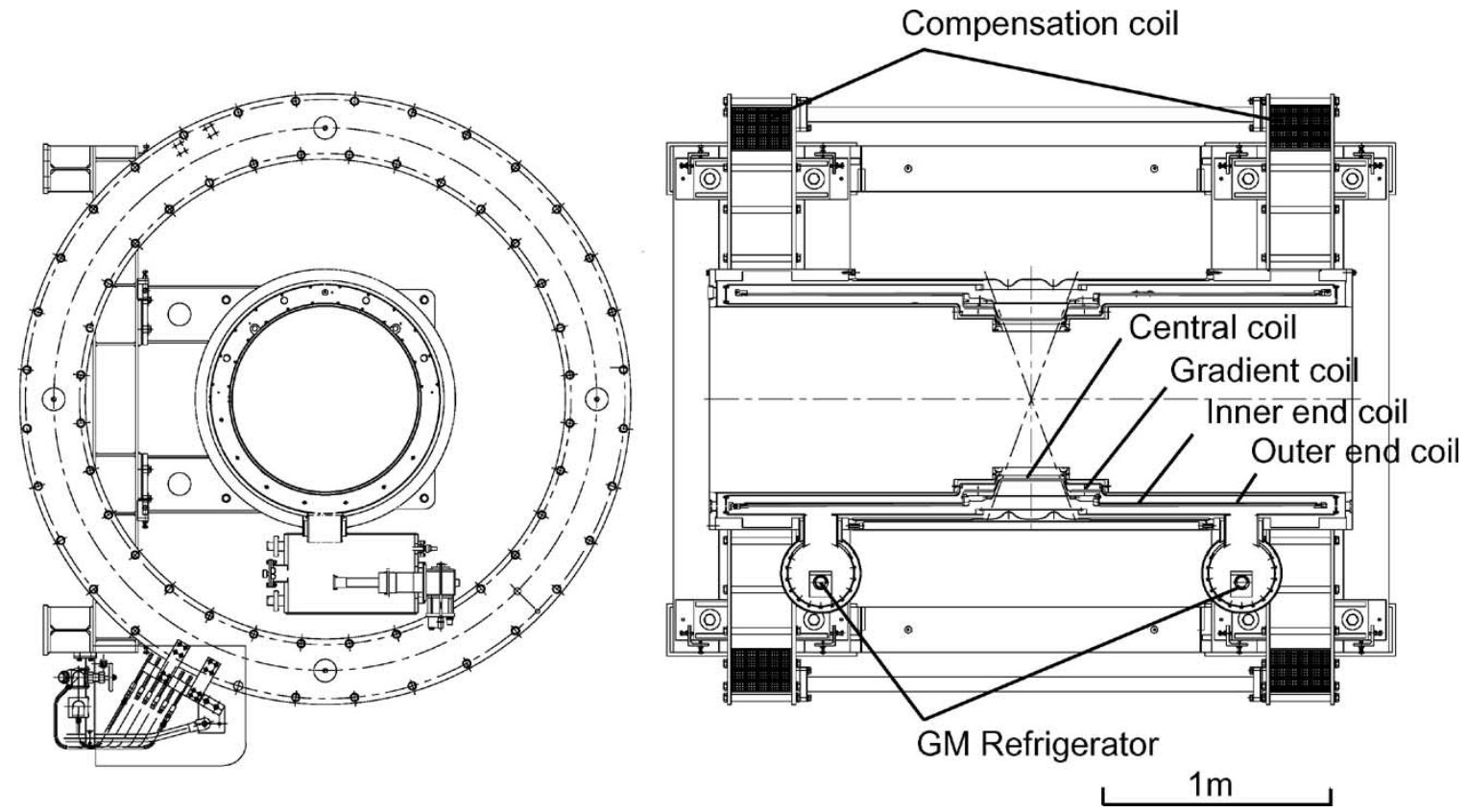

Fig. 1. A cross section of the magnet. Units in $\mathrm{mm}$.

TABLE I

PARAMETERS OF THE SUPERCONDUCTING MAGNET

\begin{tabular}{ccccc}
\hline \hline Coil & Central & Gradient & Inner end & Outer end \\
\hline Inner dia. $(\mathrm{mm})$ & 699.1 & 809.1 & 919.1 & 919.1 \\
Outer dia. $(\mathrm{mm})$ & 711.6 & 820.6 & 929.5 & 929.5 \\
Length $(\mathrm{mm})$ & 240.3 & 110.4 & 189.9 & 749.2 \\
Layers & 4 & 4 & 3 & 3 \\
Turns & 1068 & 399 & 240 & 1548 \\
Winding density (Turns $/ \mathrm{m})$ & 4444.4 & 3614.1 & 1263.8 & 2066.2 \\
Current $(\mathrm{A})$ & 360 & 360 & 360 & 360 \\
Inductance $(\mathrm{H})$ & 1.64 & 0.62 & 0.35 & 2.29 \\
Energy $E(\mathrm{~kJ})$ & 106 & 40 & 23 & 148 \\
Weight $M(\mathrm{~kg})$ & 9 & 4 & 7 & 28 \\
$E / M(\mathrm{~kJ} / \mathrm{kg})$ & 11.8 & 10.0 & 3.3 & 5.3 \\
\hline \hline
\end{tabular}
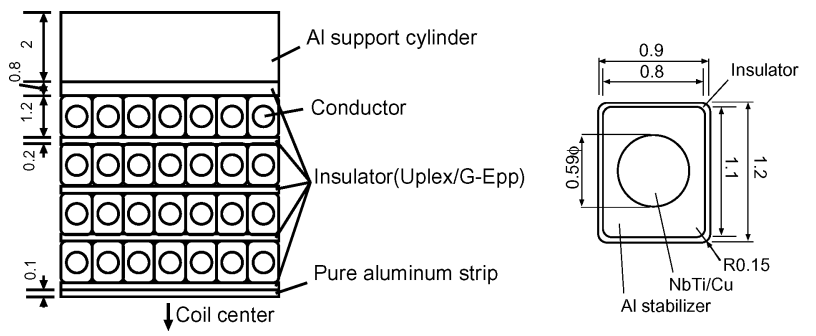

Fig. 2. Layer structure of the central coil (left) and cross section of the conductor (right). Units in $\mathrm{mm}$.

stabilizer is mechanically reinforced by means of "micro-alloying" and "cold work hardening" [2], [3]. Aluminum-stabilizer can be reinforced by adding small amount of metals such as nickel, magnesium, and copper while keeping electrical resistivity as low as possible. Nickel at $5000 \mathrm{ppm}$ is added into the aluminum-stabilizer in the conductor.

The performance of the conductor was measured around $4 \mathrm{~K}$. The overall yield strength was found to be above $220 \mathrm{MPa}$ at 4.2 K. Characteristics of superconductivity were also measured. Fig. 3 shows the measured critical current as a function of the

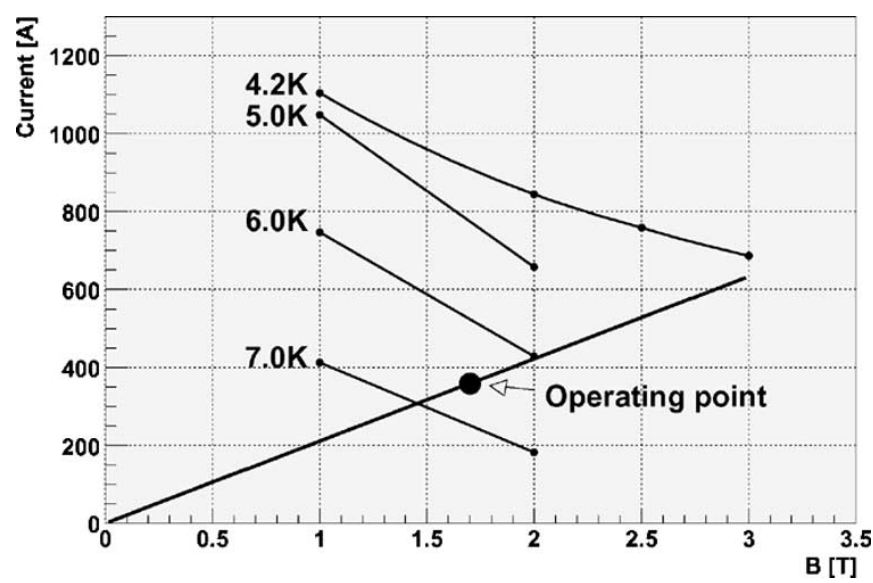

Fig. 3. Measured critical current as a function of the applied magnetic field. The load line and operating point for the magnet are also shown.

applied magnetic field. Operating current of the magnet is $360 \mathrm{~A}$ and peak value of the magnetic field is $1.7 \mathrm{~T}$ in the central coil. The load line with the operating point of the magnet is also shown in Fig. 3. This figure indicates that the performance of the conductor has a safety margin of about $40 \%$ compared to the operating condition of the magnet because the operating temperature of the coils was found to be $4.2-4.4 \mathrm{~K}$ in the excitation test which will be described in Section III.

A pair of compensation coils are implemented in the magnet in order to cancel stray magnetic field in the vicinity of the photon detector placed close to the magnet. The stray field could degrade the performance of the photon detector because the gain of the photomultiplier tube to be used in the photon detector rapidly drops as the strength of the applied magnetic field increases. The stray field should be reduced down to 50 Gauss level around the photon detector.

The polarity of the operating current for the compensation coil is the same as that for the main superconducting magnet. 


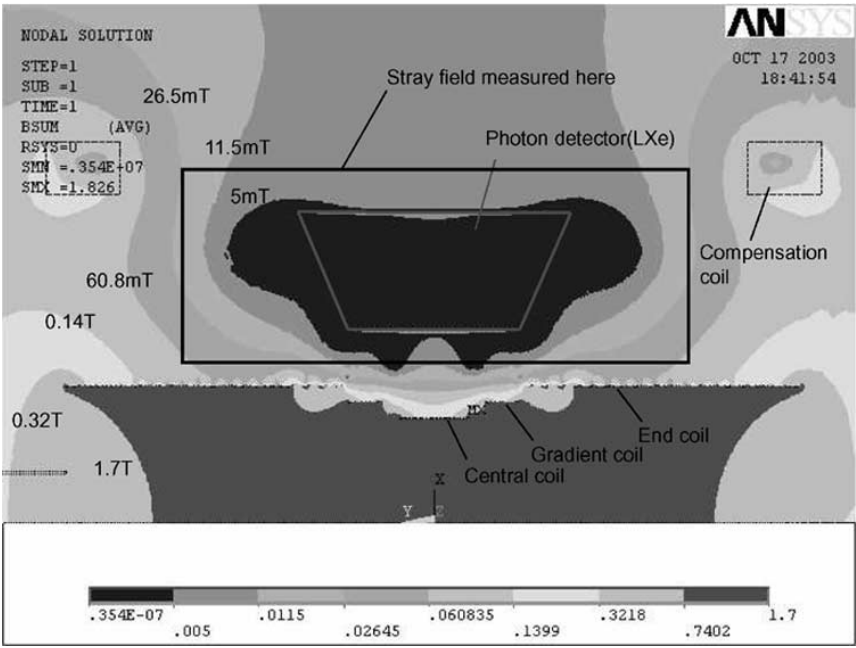

Fig. 4. Contour plot of the residual magnetic field. A box in the photon detector region shows the boundary of the photon detector. Note that the contour interval is not linear. The region shown in Fig. 4 where the stray field was measured in the excitation test is also shown.

Magnetic field around the photon detector from the main magnet can be canceled effectively by the compensation coil because the shape of the flux lines produced by the compensation coil is very similar to that produced by the main magnet in the region of the photon detector. Fig. 4 shows the calculated contour plot of the magnetic field produced by the magnet with the compensation coils. It can be seen that the residual field is very small all over the photon detector region.

\section{EXCITATION TESTS}

Excitation tests were performed to measure the performance of the magnet. The superconducting coils are cooled down mainly by the refrigerators. The liquid nitrogen cooling pipe was sometimes used to shorten the cooling time and to equalize the temperature of the coils because large temperature difference between each coil could cause undesirable thermal stress in the coils. All the coils were cooled down to $4.2-4.4 \mathrm{~K}$ in 5 days while keeping the temperature difference below $50 \mathrm{~K}$.

Temperature and stress distribution were measured during the excitation tests by using temperature sensors ( $\mathrm{Pt}, \mathrm{CGR}, \mathrm{PtCo}$ ) and strain gauges attached to the coils and support cylinders. The origin and propagation of the quench were observed by using voltage taps installed at both ends of each coil and by superconducting quench detectors (SQD's).

A series of excitation runs were performed, gradually increasing coil current. At each current step, a quench test was performed by inducing a quench by switching off the DC breaker of the power supply, firing heater or switching off the refrigerator.

The severest test was the test with a quench at the central coil at the coil current of $360 \mathrm{~A}$. In this test a quench was induced in the central coil by firing a heater. The quench was detected by the quench detector and SQD reacted at $t=130 \mathrm{msec}$ after firing the heater $(t=0)$. The protection heaters were switched on and the DC power was switched off at $t=180$ and $210 \mathrm{msec}$, respectively. The SQD's in the other coils reacted at $t=150-$ $200 \mathrm{msec}$. Fig. 5 shows the resultant voltage change across the

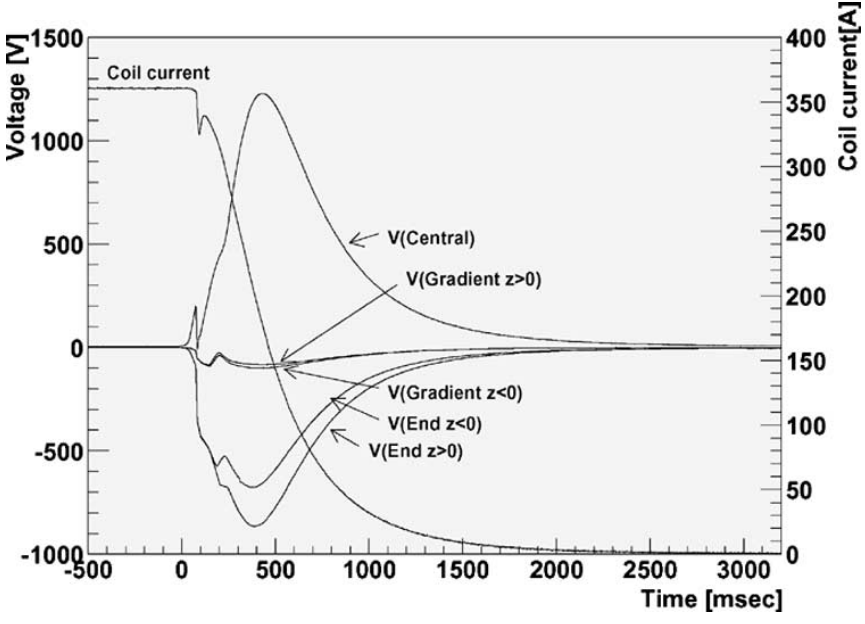

Fig. 5. Voltage change across the voltage taps in each coil in the heater quench test at $360 \mathrm{~A}$.

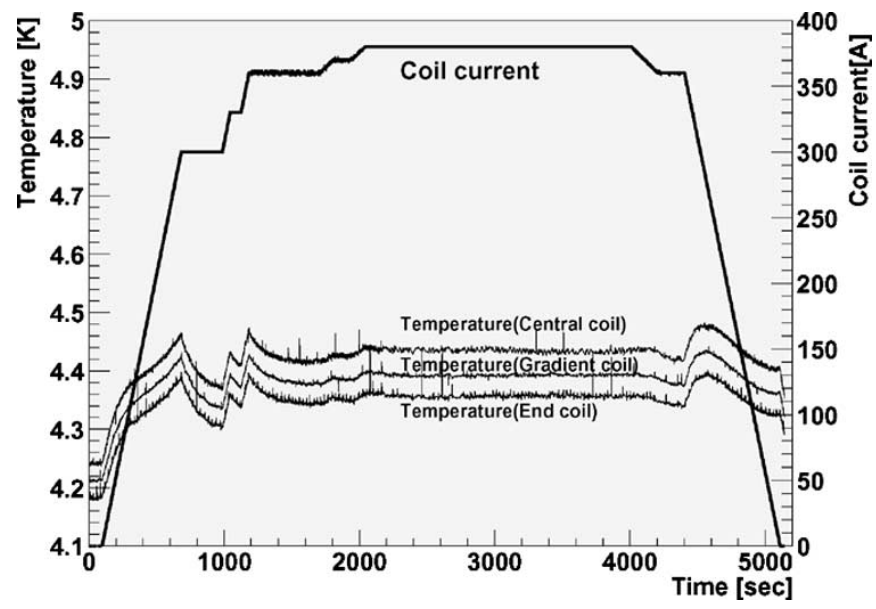

Fig. 6. Coil current and temperature change in the excitation at the coil current of $380 \mathrm{~A}$.

voltage taps at both ends of each coil. A maximum voltage rise of $1200 \mathrm{~V}$ was observed at the central coil where a quench was induced first. The temperature of the magnet also peaked in the central coil $16 \mathrm{sec}$ after the quench occurred. The maximum temperature was $110 \mathrm{~K}$.

Finally, excitation up to 380 A coil current, which is $5.6 \%$ higher than the operating current ( $360 \mathrm{~A}$ ), was performed. Fig. 6 shows the coil current as a function of time in the excitation. The temperature change of each coil is also shown in this figure. It can be seen that the temperatures of all the coils were kept below $4.5 \mathrm{~K}$ during the excitation.

The strains in the central coil and support cylinders where the axial component of the electromagnetic force concentrates were measured with strain gauges in order to verify the mechanical strength of the magnet. Fig. 7 shows change of the strains up to $380 \mathrm{~A}$ coil current as a function of square of the coil current which is proportional to the electromagnetic force acting on the magnet. This figure shows a good linear relation between the strain and square of the coil current (electromagnetic force) although slight hysteresis can be seen in some strain gauges. It indicates that mechanical strength of the coils and support structure are sufficient. 


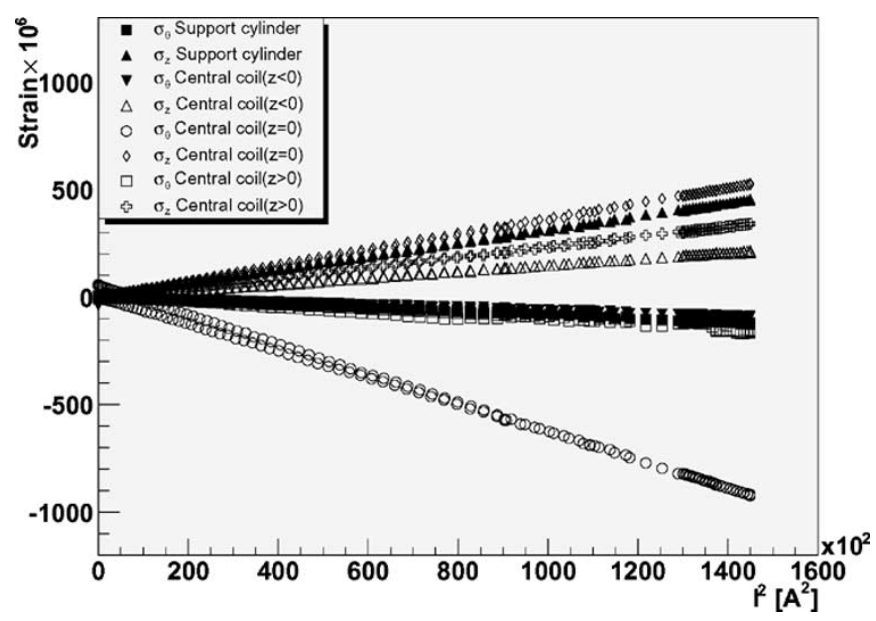

Fig. 7. Change of the strain during the excitation test at 380 A coil current.

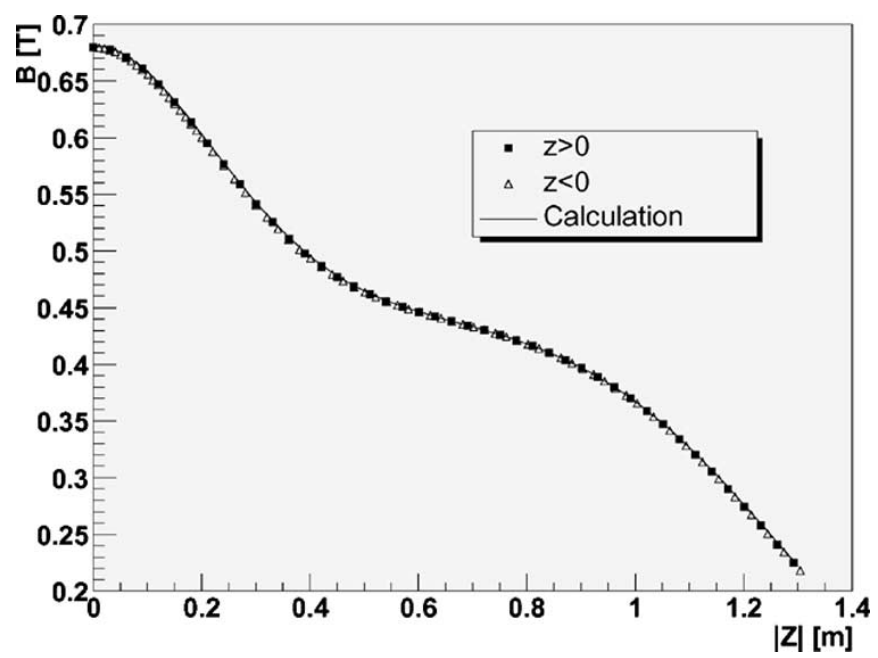

Fig. 8. Magnetic field along the magnet axis measured in the excitation test at a coil current of $200 \mathrm{~A}$. The calculated magnetic field is also shown.

The magnetic field produced in the bore of the magnet was measured. The field measurement was done at a coil current of 200 A with the compensation coils off. Fig. 8 shows the magnetic field measured along the magnet axis where calculated magnetic field is also shown. It can be seen that the magnetic field in the bore is graded as designed and shows a good agreement with the calculation.

One of the most important feature of the magnet is the cancellation of the stray magnetic field around the photon detector to be placed close to the magnet. Fig. 9 shows the stray magnetic field measured in the vicinity of the region to place photon detector as shown in Fig. 4. The measurement was done at 50\% excitation both for the main magnet and compensation coils. In this figure field strength is normalized for the full operating current for simplicity. The stray field was found to be reduced below 50 Gauss almost all over the photon detector region.

\section{CONCLUSION AND SUMmARY}

A thin-wall superconducting magnet was developed for the positron spectrometer in the MEG experiment, which is spe-

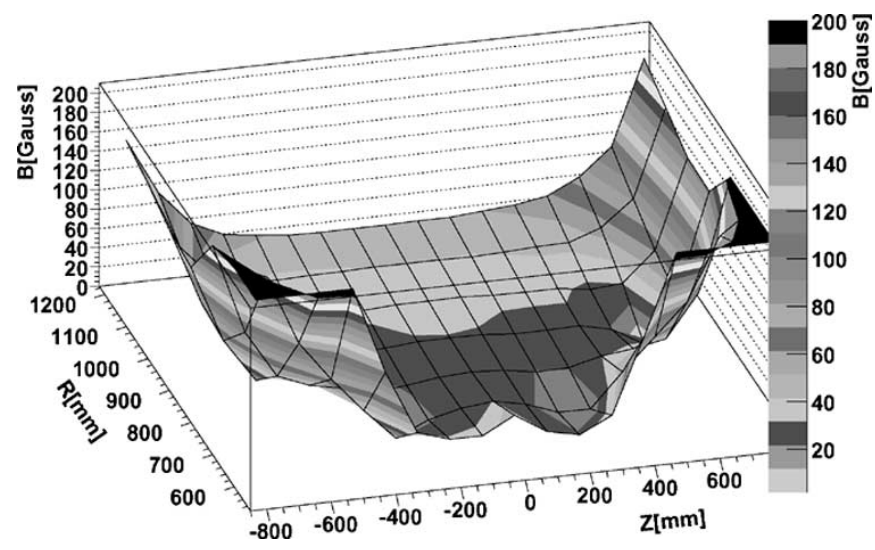

Fig. 9. Stray magnetic field measured in the photon detector region in the excitation test. The measurement was done at $50 \%$ operating current. The field strength shown in this figure is normalized to the full operating current. The region where the stray field was measured can be seen in Fig. 4 .

cially designed to provide a gradient field to achieve good features of the spectrometer. In order to minimize the thickness of the magnet, a high-strength aluminum-stabilized conductor was specially developed with micro-alloying and cold work technologies. Performance of the conductor was measured at $4 \mathrm{~K}$. It was found that the overall yield strength of the conductor above $220 \mathrm{MPa}$ at $4 \mathrm{~K}$ is achieved and the superconducting performance of the conductor has a safety margin of about $40 \%$ compared to the operating point of the magnet.

The magnet was successfully tested up to the coil current of $380 \mathrm{~A}$, which is $5.6 \%$ higher than the operating current. The quench tests were done up to $360 \mathrm{~A}$ and quench propagation was observed using voltage taps, temperature sensors and SQD's. The mechanical strength of the magnet was also measured using strain gauges. The measured magnetic field in the bore shows a good agreement with the design field. The stray magnetic field in the photon detector region was found to be less than 50 Gauss.

The results from the tests indicate that the magnet has a good performance with a reasonable margin for operation in the positron spectrometer in the MEG experiment.

\section{ACKNOWLEDGMENT}

The authors would like to thank K. Kikuchi and G. Iwaki of Hitachi Cable, Ltd. for their cooperation in the development of the conductor.

\section{REFERENCES}

[1] "Research Proposal to PSI," Available: [Online], http://meg.psi.ch, May 1999.

[2] A. Yamamoto, Y. Makida, Y. Doi, T. Kondo, K. Wada, and S. Meguro, "Development toward ultra-thin superconducting solenoid magnets for high energy particle detectors," Nucl. Phys., vol. B78, pp. 565-570, 1999.

[3] A. Yamamoto and Y. Makida et al., "A thin superconducting solenoid magnet for particle physics," IEEE Trans. Appl. Supercond., vol. 12, no. 1, pp. 438-441, March 2002.

[4] A. Dudarev et al., "Quench propagation and detection in the superconducting bus-bars of the ATLAS Magnets," IEEE Trans. on Appl. Supercond., vol. 10, no. 1, pp. 381-384, March 2000. 